\title{
2007/7
}

Quasi-variational inequality formulation of the mixed equilibrium in multiclass routing games

\author{
Laura Scrimali
}




\title{
CORE DISCUSSION PAPER
}

$2007 / 7$

\section{Quasi-variational inequality formulation of the mixed equilibrium in multiclass routing games}

\author{
Laura SCRIMALI ${ }^{1}$
}

January 2007

\begin{abstract}
In the modeling of competition on networks it is usually assumed that users either behave following the Wardrop equilibrium or the Nash equilibrium concept. Nevertheless, in several equilibrium situations, for instance in urban traffic flows, intercity freight flows and telecommunication networks, a mixed behavior is observed. This paper presents a time-dependent network model shared by two types of users: group users (Nash players) and individual users (Wardrop players). A group user has a significant impact on the load of the network, whereas an individual user has a negligible impact. Both classes of users choose the paths to ship their jobs so as to minimize their costs, but they apply different optimization criteria. The source of interaction of users is represented by the travel demand, which is assumed to be elastic with respect to the equilibrium solution. Thus, the equilibrium distribution is proved to be equivalent to the solution of an appropriate time-dependent quasivariational inequality problem. A result on the existence of solutions is discussed as well as a numerical example.
\end{abstract}

Keywords: Nash equilibrium, Wardrop equilibrium, routing, quasi-variational inequality

${ }_{1}^{1}$ Dipartimento di Matematica e Informatica, Università di Catania, Italy. E-mail: scrimali@dmi.unict.it The research presented in this paper was done at CORE.

This paper presents research results of the Belgian Program on Interuniversity Poles of Attraction initiated by the Belgian State, Prime Minister's Office, Science Policy Programming. The scientific responsibility is assumed by the author. 


\section{Introduction}

In the modeling of competition on networks it is usually assumed that users either behave following the Wardrop equilibrium or the Nash equilibrium concept. Nevertheless, in several equilibrium situations a mixed behavior is observed. We mention, for instance, the case of intercity freight flows, in which one recognizes the presence of shippers who have different market power. Thus, the mixed equilibrium appears as the most natural and appropriate equilibrium concept, which is able to represent a situation in which larger shippers aim at minimizing their own costs, acting via the system equilibrium concept, whereas smaller shippers are user-equilibrium oriented. An analogous situation may occur in telecommunication networks, where different competitive forces act in the market.

This paper presents time-dependent multiclass routing games which occur in telecommunication networks (see $[1,4,9,20]$ for some relevant results on this topic). We assume that the network model is shared by two types of users: group users (Nash players or player with market power) and individual users (Wardrop players or price-takers). A group user has a significant impact on the load of the network, whereas an individual user has a negli-

gible impact. Both classes of users choose the routes to ship their jobs so as to minimize their costs, but they follow their own optimization criteria.

Our aim is to characterize time-dependent equilibria in this mixed situation and provide an opportune variational formulation. As we are interested in analyzing and describing time-dependent phenomena, we allow all data to be time-specific. Moreover, we assume that the amount of jobs spread through the network is bounded and users incur in equilibrium-dependent travel demands. Thus, the time-dependent mixed equilibrium model is described by an infinite-dimensional quasi-variational inequality problem of the form:

$$
\text { Find } x^{*} \in K\left(x^{*}\right): \quad \int_{0}^{T}\left\langle F\left(t, x^{*}(t)\right), x(t)-x^{*}(t)\right\rangle d t \geq 0, \quad \forall x(t) \in K\left(x^{*}\right),
$$

where $F:[0, T] \times \mathbb{R}^{n+w} \rightarrow \mathbb{R}^{n+w}, K: E \rightarrow 2^{L^{2}\left(0, T ; \mathbb{R}^{n+w}\right)}, E$ is a nonempty, convex, bounded and closed subset of $L^{2}\left(0, T ; \mathbb{R}^{n+w}\right)$ and $n, w \in \mathbb{N}$ will be

mento di Matematica e Informatica, Università di Catania, Viale Andrea Doria 6, 95125 Catania, Italy; e-mail: scrimali@dmi.unict.it.

This text presents research results of the Belgian Program on Interuniversity Poles of Attraction initiated by the Belgian State, Prime Minister's Office, Science Policy Programming. The scientific responsibility is assumed by the author. 
specified in the following. We are concerned with providing conditions for the existence of solutions to such a problem. We address the interested reader to the monograph [2] and references therein for a survey of theory and applications of quasi-variational inequalities.

Research presented in this paper advances the modeling of routing games in the following ways:

1. We extend the mixed behavior traffic network equilibrium model (see [9] and the recent work [4]) as we take into consideration the evolution of the system with respect to time. Thus, we do not refer to an instantaneous network situation, but we focus on the actual network situation and take into account all the travel times experienced by users during the time interval of interest. In addition, we take into consideration capacity constraints on the multiflows spread through the network, thus improving the unconstrained models known in the literature.

2. The source of interaction of users is represented by travel demands which are assumed to be elastic with respect to the equilibrium solution. This allows us to capture all the equilibria adjustment processes. As a consequence, the problem is expressed as an infinite-dimensional quasi-variational inequality problem.

3. As already realized in [9], unlike the standard market oligopoly model, the mixed behavior equilibrium model contains some externalities so that some paradoxical results may be observed. This outcome deserves more attention and may unveil new features of network-based competition models.

The paper is organized as follows. In Section 2 a detailed description of the network system is given and the mixed equilibrium concept is presented. In Section 3 a complete characterization of mixed equilibria in terms of an opportune quasi-variational inequality is given. In Section 4 we provide an existence result, followed, in Section 5, by a numerical example. Section 6 summarizes our findings and presents some further research issues.

\section{Mixed network equilibrium}

In the time interval $[0, T]$ we consider a general network $G(\mathcal{M}, \mathcal{L})$, where $\mathcal{M}$ is the set of nodes and $\mathcal{L}$ is the set of directed links through the nodes. 
As we are interested in a time-dependent setting, our functional space is the Hilbert space $L^{2}\left(0, T ; \mathbb{R}^{q}\right)$ (with $T>0$ ) of square-integrable functions from the closed interval $[0, T]$ to $\mathbb{R}^{q}$ ( $q$ will be specified in the following), endowed with the scalar product $\langle\cdot, \cdot\rangle_{L^{2}}=\int_{0}^{T}\langle\cdot, \cdot\rangle d t$ and the usual associated norm $\|\cdot\|_{L^{2}}$. As usual the scalar product in $\mathbb{R}^{q}$ is denoted by $\langle\cdot, \cdot\rangle$ and the norm by $\|\cdot\|$. We present a path-based model, however, sometimes (see numerical example in Section 5), the link-based model will be considered. Therefore, we introduce two notations, one in terms of paths and the other in terms of links (see model description in [4]).

We focus on a network shared by two types of users: group users and individual users. We denote by $\mathcal{N}$ the set of group users, with $|\mathcal{N}|=N(|A|$ denotes the number of elements of the set $A$ ) and by $\mathcal{W},|\mathcal{W}|=W$, the set of classes of individual users. We denote by $\mathcal{I}=\mathcal{N} \cup \mathcal{W}$ the set of all users in the network and assume that $\mathcal{I}$ is finite. It results $|\mathcal{I}|=I=N+W$.

Each user $i \in \mathcal{I}$ has to ship some jobs from a source $s$ to a destination $d$ with a service rate at time $t$ denoted by $\mu^{i}(t)$. We set $\mu^{i}(t)=\left(\mu_{a}^{i}(t), a=\right.$ $1, \ldots, L)$, where $L=|\mathcal{L}|$. The service rate $\mu_{a}^{i}(t)$ may be regarded as the speed at which the job routed by class $i$ is processed at link $a$ at time $t$. We also assume that $\mu_{a}^{i}(t)>0$, for a.e. $t \in[0, T], \forall a \in \mathcal{L}$. For each user $i$ we denote by $\delta_{(s, d)}^{i}(t)$ the travel demand for the jobs leaving the source of the origin-destination pair $(s, d)$ at time $t$.

Individual users are grouped into classes and also a group user is called a class, see reference [4]. In addition, there are numerous classes of jobs, each of which represents a large number of jobs. In the context of road traffic, for instance, an individual user may correspond to a single driver, and the class may correspond to all the drivers of a given type of vehicle that have a given source and destination; a group user may be a transportation company.

A group user has a significant impact on the load of the network and, as a consequence, on delays in which any other user may incur. This type of user follows a class-centralized optimization approach, in the sense that routing decisions are taken by a single decision maker and, hence, the appropriate equilibrium concept is the Nash equilibrium. Clearly, when all origin-destination pairs are controlled by a single decision maker, the model results in the well-known system equilibrium. If this extreme case is not verified, each class of group users exhibits a system equilibrium-like behavior, but the system is not in a system equilibrium.

Each user $i \in \mathcal{N}$ is characterized by:

- one service rate $\mu^{i}(t)=\left(\mu_{a}^{i}(t)\right)_{a \in \mathcal{L}}$; 
- a set of origin-destination pairs $D^{i}$;

- a travel demand vector $\delta^{i}(t)=\left(\delta_{(s, d)}^{i}(t)\right)_{(s, d) \in D^{i}}$.

Individual users, whose routing decisions have a negligible impact on the load of the network, have a single job to route from a given source to a given destination with a given service rate.

Each user $i \in \mathcal{W}$ is characterized by:

- one service rate $\mu^{i}(t)=\left(\mu_{a}^{i}(t)\right)_{a \in \mathcal{L}}$;

- one origin-destination pair $(s, d)$;

- one travel demand $\delta_{(s, d)}^{i}(t)$.

We remark that all the jobs of class $i$ have the same service rate.

We now introduce some other network customary concepts. We denote by $p$ a sequence of directed links from a source to a destination. For $i \in \mathcal{I}$ we denote by $\mathcal{P}^{i}$ the set of paths used by class $i$, by $\mathcal{P}_{(s, d)}^{i}$ the set of paths used by class $i$ from source $s$ to destination $d$. We also have $\mathcal{P}^{i}=\cup_{(s, d) \in D^{i}} \mathcal{P}_{(s, d)}^{i}$ and $\mathcal{P}=\cup_{i \in \mathcal{I}} \mathcal{P}^{i}$. Let $n=\sum_{i \in \mathcal{N}}\left|\mathcal{P}^{i}\right|$ be the number of paths used by Nash players, $w=\sum_{i \in \mathcal{W}}\left|\mathcal{P}^{i}\right|$ the number of paths used by Wardrop players and let $n+w=\sum_{i \in \mathcal{I}}\left|\mathcal{P}^{i}\right|$ be the total number of paths in the network.

For $i \in \mathcal{I}$ and $a \in \mathcal{L}$, let $f_{a}^{i}(t)$ the amount of jobs sent by class $i$ and circulating on link $a$ at time $t$. For $i \in \mathcal{I}$ we group $f_{a}^{i}(t)$ into the vector $f^{i}(t)=$ $\left(f_{1}^{i}(t), \ldots, f_{L}^{i}(t)\right)^{T}$. Then, we denote by $f_{a}(t)=\sum_{i \in \mathcal{N}} f_{a}^{i}(t)+\sum_{i \in \mathcal{W}} f_{a}^{i}(t)$ the total flow on link $a$. Finally, we set $f(t)=\left(f_{1}(t), \ldots, f_{L}(t)\right)^{T}$, which represents the link strategy vector.

Analogously, for $i \in \mathcal{I}$ and $p \in \mathcal{P}^{i}$, let $x_{p}^{i}(t)$ the amount of jobs sent by class $i$ and circulating on path $p$ at time $t$. We remark that if $i \in \mathcal{N}$, $x_{p}^{i}(t)$ is the amount of jobs of user $i$ sent through path $p$, whereas, if $i \in \mathcal{W}, x_{p}^{i}(t)$ represents the amount of individual users of class $i$ that choose path $p$ to ship their unique job. We group the amounts of jobs $x_{p}^{i}(t)$ into the vector $x^{i}(t)=\left(x_{1}^{i}(t), \ldots, x_{\left|\mathcal{P}^{i}\right|}^{i}(t)\right)^{T}$ and further into the vector $x(t)=$ $\left(x^{1}(t), \ldots, x^{I}(t)\right)^{T}$, which represents the path strategy vector. We may also write $x(t)=\left(x^{\mathcal{N}}(t), x^{\mathcal{W}}(t)\right)^{T}$, where $x^{\mathcal{N}}(t)=\left(x^{1}(t), \ldots, x^{N}(t)\right)^{T}$ and $x^{\mathcal{W}}(t)=\left(x^{1}(t), \ldots, x^{W}(t)\right)^{T}$. Moreover, we may set $x(t)=\left(x_{p}^{i}(t)\right)_{i \in \mathcal{I}, p \in \mathcal{P}^{i}}$, namely $x(t)$ may be regarded as the $n+w$-dimensional multiflow generated on paths by all the users of the network.

We denote by $\Gamma$ is the link-path incidence matrix, whose typical entry $\gamma_{a p}$ is 1 if link $a$ is contained in path $p$ and 0 otherwise. It then results 
that $f_{a}^{i}(t)=\sum_{p \in \mathcal{P}^{i}} \gamma_{a p} x_{p}^{i}(t), \forall i \in \mathcal{I}$. Finally, $\forall a \in \mathcal{L}$ and $\forall i \in \mathcal{I}$, we introduce the link cost functions $c_{a}^{i}(f(t))$. We group $c_{a}^{i}(f(t))$ into the vector $c^{i}(f(t))=\left(c_{1}^{i}(f(t)), \ldots, c_{L}^{i}(f(t))\right)^{T}$ and further into the vector $c(t)=$ $\left(c^{1}(f(t)), \ldots, c^{I}(f(t))\right)^{T}$, which represents the link strategy vector.

In the following, we assume that user $i \in \mathcal{I}$ incurs in elastic travel demands which depend on the equilibrium distribution $x^{*}(t)$. Let $m_{\mathcal{N}}=$ $\sum_{i \in \mathcal{N}}\left|D^{i}\right|$ the number of $\mathrm{O} / \mathrm{D}$ pairs controlled by Nash users and $m=$ $m_{\mathcal{N}}+1=\sum_{i \in \mathcal{I}}\left|D^{i}\right|$ the total number of $\mathrm{O} / \mathrm{D}$ pairs in the network. Thus, we may introduce the travel demand function $\delta(t, x):[0, T] \times \mathbb{R}^{n+w} \rightarrow \mathbb{R}^{m}$ such that

a) $\delta(t, x)$ is measurable in $t \forall x \in \mathbb{R}^{n+w}$, continuous in $x$ for a.e. $t \in[0, T]$, and

$$
\exists \gamma_{1} \in L^{2}(0, T), \eta_{1}(t) \in L^{\infty}(0, T):\|\delta(t, x)\| \leq \gamma_{1}(t)+\eta_{1}(t)\|x\| .
$$

Now, let $E$ be a nonempty, convex, bounded and closed subset of $L^{2}\left(0, T ; \mathbb{R}^{n+w}\right)$ and let $K$ be the multifunction $K: E \rightarrow 2^{L^{2}\left(0, T ; \mathbb{R}^{n+w}\right)}$ defined as

$$
\begin{aligned}
K\left(x^{*}\right)= & \left\{x(t) \in L^{2}\left(0, T ; \mathbb{R}^{n+w}\right): \forall i \in \mathcal{I}, \forall(s, d) \in D^{i}, \forall p \in \mathcal{P}_{(s, d)}^{i},\right. \\
& \left.0 \leq x_{p}^{i}(t) \leq \bar{x}_{p}^{i}(t), \sum_{p \in \mathcal{P}_{(s, d)}^{i}} x_{p}^{i}(t)=\delta_{(s, d)}^{i}\left(t, x^{*}(t)\right) \text { a.e. } t \in[0, T]\right\},
\end{aligned}
$$

where $\bar{x}_{p}^{i}(t), i \in \mathcal{I}, p \in \mathcal{P}^{i}$ is the upper bound on the amount of job sent through path $p$ by user $i$ at time $t$.

Remark 1 It results that $K\left(x^{*}\right)=K^{\mathcal{N}}\left(x^{*}\right) \times K^{\mathcal{W}}\left(x^{*}\right)$, where

$$
\begin{aligned}
K^{\mathcal{N}}\left(x^{*}\right)= & \left\{x^{\mathcal{N}}(t) \in L^{2}\left(0, T ; \mathbb{R}^{N}\right): \forall i \in \mathcal{N}, \forall(s, d) \in D^{i}, \forall p \in \mathcal{P}_{(s, d)}^{i},\right. \\
& \left.0 \leq x_{p}^{i}(t) \leq \bar{x}_{p}^{i}(t), \sum_{p \in \mathcal{P}_{(s, d)}^{i}} x_{p}^{i}(t)=\delta_{(s, d)}^{i}\left(t, x^{*}(t)\right) \text { a.e. } t \in[0, T]\right\},
\end{aligned}
$$

and

$$
\begin{aligned}
K^{\mathcal{W}}\left(x^{*}\right)= & \left\{x^{\mathcal{W}}(t) \in L^{2}\left(0, T ; \mathbb{R}^{W}\right): \forall i \in \mathcal{W}, \forall p \in \mathcal{P}_{(s, d)}^{i}, 0 \leq x_{p}^{i}(t) \leq \bar{x}_{p}^{i}(t),\right. \\
& \left.\sum_{p \in \mathcal{P}_{(s, d)}^{i}} x_{p}^{i}(t)=\delta_{(s, d)}^{i}\left(t, x^{*}(t)\right) \text { a.e. } t \in[0, T]\right\} .
\end{aligned}
$$


We now introduce the cost functions for the classes of users. We denote by

$$
J^{i}(t, x):[0, T] \times \mathbb{R}^{n+w} \rightarrow \mathbb{R}_{+}
$$

the cost function of class $i \in \mathcal{N}$, and by

$$
C_{p}^{i}(t, x):[0, T] \times \mathbb{R}^{n+w} \rightarrow \mathbb{R}_{+}
$$

the cost function on path $p$ for each user of class $i \in \mathcal{W}$.

In order to present a compact formulation, we introduce the notations

$$
\begin{aligned}
(J(t, x))^{T} & =\left(J^{i}(t, x)\right)_{i \in \mathcal{N}} \in \mathbb{R}^{N}, \\
C(t, x) & =\left(C_{p}^{i}(t, x)\right)_{i \in \mathcal{W}, p \in \mathcal{P}^{i}} \in \mathbb{R}^{w} .
\end{aligned}
$$

The following assumptions will be required:

b) $J(t, x)$ is measurable in $t \forall x \in \mathbb{R}^{n+w}$, convex and continuously differentiable with respect to $x$ for a.e. $t \in[0, T]$, and

$$
\exists \gamma_{2} \in L^{1}(0, T), \eta_{2}(t) \in L^{\infty}(0, T):\|J(t, x)\| \leq \gamma_{2}(t)+\eta_{2}(t)\|x\|^{2} ;
$$

c) $C(t, x)$ is measurable in $t \forall x \in \mathbb{R}^{n+w}$, continuous in $x$ for a.e. $t \in[0, T]$, and

$$
\exists \gamma_{3} \in L^{2}(0, T), \eta_{3}(t) \in L^{\infty}(0, T):\|C(t, x)\| \leq \gamma_{3}(t)+\eta_{3}(t)\|x\| .
$$

For every $i \in \mathcal{N}$ let $\Psi_{p}^{i}(t, x)$ be the derivative of $J^{i}(t, x)$ with respect to $x_{p}^{i}$. Then it result $\Psi(t, x)=\left(\Psi_{p}^{i}(t, x)\right)_{i \in \mathcal{N}, p \in \mathcal{P}^{i}} \in \mathbb{R}^{n}$. We suppose that

d) $\Psi(t, x)$ is measurable in $t \forall x \in \mathbb{R}^{n+w}$, continuous in $x$ for a.e. $t \in[0, T]$, and

$$
\exists \gamma_{4} \in L^{2}(0, T), \eta_{4}(t) \in L^{\infty}(0, T):\|\Psi(t, x)\| \leq \gamma_{4}(t)+\eta_{4}(t)\|x\| .
$$

Users aim at minimizing their costs according to different criteria. The optimization problem of user $i \in \mathcal{N}$ is the following minimization problem in which all other players's strategies are assumed as fixed:

$$
i \in \mathcal{N}, \quad \min _{\substack{x^{i}(t) \text { s.t. } \\\left(x^{*-i}(t), x^{i}(t)\right) \in K\left(x^{*}\right)}} \int_{0}^{T} J^{i}\left(t, x^{*-i}(t), x^{i}(t)\right) d t .
$$

The functional $J^{i}(t, x(t))$ is convex in $x$ and, by assumption $\mathrm{b}$ ), it belongs to $L^{1}(0, T)$ and is continuous therein. Moreover, $K\left(x^{*}\right)$, as will be proved in Theorem 4.2, is a convex, bounded and closed subset of the Hilbert space $L^{2}\left(0, T ; \mathbb{R}^{n+w}\right)$ and therefore it is also weakly compact (see Theorem B.4 in [10]). By Lemma 2.11 and Theorem 2.3 in [10], a solution to (1) does exist. 
Remark 2 If $K\left(x^{*}\right)=\prod_{i \in \mathcal{I}} K^{i}\left(x^{*}\right)$, where for all $i \in \mathcal{I}$

$$
\begin{aligned}
K^{i}\left(x^{*}\right)= & \left\{x^{i}(t) \in L^{2}\left(0, T ; \mathbb{R}^{\left|\mathcal{P}^{i}\right|}\right): \forall(s, d) \in D^{i}, \forall p \in \mathcal{P}_{(s, d)}^{i},\right. \\
& \left.0 \leq x_{p}^{i}(t) \leq \bar{x}_{p}^{i}(t), \sum_{p \in \mathcal{P}_{(s, d)}^{i}} x_{p}^{i}(t)=\delta_{(s, d)}^{i}\left(t, x^{*}(t)\right) \text { a.e. } t \in[0, T]\right\},
\end{aligned}
$$

then minimization problem (1) becomes

$$
i \in \mathcal{N}, \quad \min _{x^{i}(t) \in K^{i}\left(x^{*}(t)\right)} \int_{0}^{T} J^{i}\left(t, x^{*-i}(t), x^{i}(t)\right) d t .
$$

User $i \in \mathcal{W}$, who takes costs as given, fulfills a Wardrop-type principle. However, due to the presence of capacity constraints, we can not refer to the usual Wardrop principle (see [19]), hence we have recourse to a generalized form (see [14]), which is able to represent the constrained network model.

We are now ready to provide the mixed equilibrium definition.

Definition $1 x^{*}(t) \in K\left(x^{*}\right)$ is a mixed equilibrium if, for a.e. $t \in[0, T]$, the subsequent conditions hold

$$
\begin{gathered}
\forall i \in \mathcal{N}, \forall(s, d) \in D^{i}, \forall p \in \mathcal{P}_{(s, d)}^{i} \\
\Psi_{p}^{i}\left(t, x^{*}(t)\right)+\alpha_{p}^{i}(t)-\beta^{i}(t) \begin{cases}\geq 0 & \text { if } x_{p}^{* i}(t)=0 \\
=0 & \text { if } 0<x_{p}^{* i}(t)<\bar{x}_{p}^{i}(t) \\
\leq 0 & \text { if } x_{p}^{* i}(t)=\bar{x}_{p}^{i}(t) ;\end{cases} \\
\left(\Psi_{p}^{i}\left(t, x^{*}(t)\right)-\beta^{i}(t)\right)\left(\bar{x}_{p}^{i}(t)-x_{p}^{* i}(t)\right)=0 ;
\end{gathered}
$$

$\forall i \in \mathcal{W}, \forall p, q \in \mathcal{P}_{(s, d)}^{i}$,

$$
C_{p}^{i}\left(t, x^{*}(t)\right)<C_{q}^{i}\left(t, x^{*}(t)\right) \Rightarrow x_{p}^{* i}(t)=\bar{x}_{p}^{* i}(t) \text { or } x_{q}^{* i}(t)=0,
$$

where $\alpha_{p}^{i}(t) \in L^{2}(0, T)$ and $\beta^{i}(t)=\beta_{(s, d)}^{i}(t) \in L^{2}(0, T)$ are the Lagrange functions associated with bound constraints and demand requirements, respectively. They are not known a priori, however, as will be seen in Theorem 3.1 and Theorem 3.2, conditions (3), (4) and (5) lead to a quasi-variational inequality in which no such functions appear. 


\section{The quasi-variational inequality formulation}

In this section, we give a complete characterization of the mixed equilibrium as a solution to a suitable quasi-variational inequality. We start with proving some preliminary results.

Theorem $1 x^{* i}(t), i \in \mathcal{N}$, is a solution to (1) if and only if it solves the problem

$$
\begin{aligned}
& \int_{0}^{T} \sum_{p \in \mathcal{P}^{i}} \Psi_{p}^{i}\left(t, x^{*}(t)\right)\left(x_{p}^{i}(t)-x_{p}^{* i}(t)\right) d t \geq 0, \\
& \forall x^{i}(t):\left(x^{1}(t), \ldots, x^{i}(t), \ldots, x^{N}(t)\right) \in K^{\mathcal{N}}\left(x^{*}\right) .
\end{aligned}
$$

Proof. The proof uses standard arguments of variational inequality theory and network equilibrium problems (see $[6,7,8,17]$ ) and therefore will be omitted.

Theorem $2 x^{* i}(t), i \in \mathcal{N}$, is a solution to (1) or (6) if and only if it satisfies conditions (3) and (4).

Proof. The proof is performed applying arguments of Lagrangian theory. For $i \in \mathcal{N}$ let us consider the function

$$
\begin{aligned}
\mathcal{L}\left(x^{i}(t), \alpha^{i}(t), \beta^{i}(t)\right)= & \int_{0}^{T} \sum_{p \in \mathcal{P}^{i}} \Psi_{p}^{i}\left(t, x^{*}(t)\right)\left(x_{p}^{i}(t)-x_{p}^{* i}(t)\right) d t \\
& -\int_{0}^{T} \sum_{p \in \mathcal{P}^{i}} \alpha_{p}^{i}(t)\left(\bar{x}_{p}^{i}(t)-x_{p}^{i}(t)\right) d t \\
& -\int_{0}^{T} \beta^{i}(t)\left(\sum_{p \in \mathcal{P}_{(s, d)}^{i}} x_{p}^{i}(t)-\delta_{(s, d)}^{i}\left(t, x^{*}(t)\right)\right) d t
\end{aligned}
$$

where $x(t) \in L^{2}\left(0, T ; \mathbb{R}^{n+w}\right)$ and

$$
\begin{aligned}
\left(\alpha^{i}(t), \beta^{i}(t)\right) \in & \mathcal{C} \\
\mathcal{C}= & \left\{\alpha^{i}(t) \in L^{2}\left(0, T ; \mathbb{R}^{\left|\mathcal{P}^{i}\right|}\right), \alpha_{p}^{i}(t) \geq 0, p \in \mathcal{P}^{i}\right. \\
& \left.\beta^{i}(t) \in L^{2}(0, T), i=1, \ldots, N\right\}
\end{aligned}
$$


By applying arguments of Lagrangian theory (see $[5,10]$ ), it is possible to prove that there exist $\alpha_{p}^{i}(t) \geq 0, p \in \mathcal{P}^{i}$ and

$$
\begin{aligned}
& \int_{0}^{T} \sum_{p \in \mathcal{P}^{i}} \alpha_{p}^{i}(t)\left(\bar{x}_{p}^{i}(t)-x_{p}^{* i}(t)\right) d t=0 \Rightarrow \alpha_{p}^{i}(t)\left(\bar{x}_{p}^{i}(t)-x_{p}^{* i}(t)\right)=0 \\
& p \in \mathcal{P}^{i}, \text { a.e. in }[0, T] .
\end{aligned}
$$

Moreover, characterizing solutions as saddle points we may write

$$
\begin{aligned}
& \mathcal{L}\left(x^{i}(t), \alpha^{i}(t), \beta^{i}(t)\right) \\
& =\int_{0}^{T} \sum_{p \in \mathcal{P}^{i}}\left(\Psi_{p}^{i}\left(t, x^{*}(t)\right)+\alpha_{p}^{i}(t)-\beta^{i}(t)\right)\left(x_{p}^{i}(t)-x_{p}^{* i}(t)\right) d t \geq 0,
\end{aligned}
$$

$\forall x(t) \in L^{2}\left(0, T ; \mathbb{R}^{n+w}\right)$. Let us suppose that $x_{q}^{i}(t)=x_{q}^{* i}(t), \forall q \neq p$, hence we get

$$
\begin{aligned}
& \int_{0}^{T} \sum_{p \in \mathcal{P}^{i}}\left(\Psi_{p}^{i}\left(t, x^{*}(t)\right)+\alpha_{p}^{i}(t)-\beta^{i}(t)\right)\left(x_{p}^{i}(t)-x_{p}^{* i}(t)\right) d t \\
& =\int_{0}^{T}\left(\Psi_{p}^{i}\left(t, x^{*}(t)\right)+\alpha_{p}^{i}(t)-\beta^{i}(t)\right)\left(x_{p}^{i}(t)-x_{p}^{* i}(t)\right) d t \geq 0 .
\end{aligned}
$$

In order to prove condition (3), we consider the following cases:

1. If $x_{p}^{* i}(t)=0$ for a.e. $t \in[0, T]$, we show that

$$
\Psi_{p}^{i}\left(t, x^{*}(t)\right)+\alpha_{p}^{i}(t)-\beta^{i}(t) \geq 0 .
$$

We argue by contradiction and suppose that there exists a set of positive measure $\Omega \subset[0, T]$ so that $\Psi_{p}^{i}\left(t, x^{*}(t)\right)+\alpha_{p}^{i}(t)-\beta^{i}(t)<0$ for a.e. $t \in \Omega$. Let us choose $x_{p}^{i}(t)$ as follows

$$
x_{p}^{i}(t) \begin{cases}>0 & \text { if } t \in \Omega \\ =x_{p}^{* i}(t) & \text { if } t \in[0, T] \backslash \Omega,\end{cases}
$$

then expression (8) results in

$$
\begin{aligned}
& \int_{0}^{T}\left(\Psi_{p}^{i}\left(t, x^{*}(t)\right)+\alpha_{p}^{i}(t)-\beta^{i}(t)\right)\left(x_{p}^{i}(t)-x_{p}^{* i}(t)\right) d t \\
& =\int_{\Omega}\left(\Psi_{p}^{i}\left(t, x^{*}(t)\right)+\alpha_{p}^{i}(t)-\beta^{i}(t)\right) x_{p}^{i}(t) d t<0 .
\end{aligned}
$$


2. If $x_{p}^{* i}(t)=\bar{x}_{p}^{i}(t)$ for a.e. $t \in[0, T]$, we prove that

$$
\Psi_{p}^{i}\left(t, x^{*}(t)\right)+\alpha_{p}^{i}(t)-\beta^{i}(t) \leq 0 .
$$

We argue by contradiction and consider a set of positive measure $\Omega \subset$ $[0, T]$ so that $\Psi_{p}^{i}\left(t, x^{*}(t)\right)+\alpha_{p}^{i}(t)-\beta^{i}(t)>0$ for a.e. $t \in \Omega$. By choosing $x_{p}^{i}(t)$ as follows

$$
x_{p}^{i}(t) \begin{cases}<\bar{x}_{p}^{i}(t) & \text { if } t \in \Omega \\ =x_{p}^{* i}(t) & \text { if } t \in[0, T] \backslash \Omega,\end{cases}
$$

it results that

$$
\begin{aligned}
& \int_{0}^{T}\left(\Psi_{p}^{i}\left(t, x^{*}(t)\right)+\alpha_{p}^{i}(t)-\beta^{i}(t)\right)\left(x_{p}^{i}(t)-x_{p}^{* i}(t)\right) d t \\
& =\int_{\Omega}\left(\Psi_{p}^{i}\left(t, x^{*}(t)\right)+\alpha_{p}^{i}(t)-\beta^{i}(t)\right)\left(x_{p}^{i}(t)-\bar{x}_{p}^{i}(t)\right) d t<0 .
\end{aligned}
$$

3. Finally, if we suppose that $0<x_{p}^{* i}(t)<\bar{x}_{p}^{i}(t)$ for a.e. $t \in[0, T]$, by using the same technique as in the previous cases, it can be easily proved that $\Psi_{p}^{i}\left(t, x^{*}(t)\right)+\alpha_{p}^{i}(t)-\beta^{i}(t)$ can not be either positive or negative on any set of positive measure.

In order to prove condition (4) we proceed as follows. If $x_{p}^{* i}(t)=\bar{x}_{p}^{i}(t)$ a.e in $[0, T]$ or $0<x_{p}^{* i}(t)<\bar{x}_{p}^{i}(t)$ a.e in $[0, T]$, condition (4) immediately follows from (3). We now suppose that $x_{p}^{* i}(t)=0$ a.e in $[0, T]$. We set

$$
\lambda(t)=\Psi_{p}^{i}\left(t, x^{*}(t)\right)+\alpha_{p}^{i}(t)-\beta^{i}(t),
$$

and choosing

$$
x_{p}^{i}(t)=\lambda(t),
$$

inequality (8) becomes

$$
\int_{0}^{T} \sum_{p \in \mathcal{P}^{i}}\left(\Psi_{p}^{i}\left(t, x^{*}(t)\right)+\alpha_{p}^{i}(t)-\beta^{i}(t)\right)^{2} d t \geq 0 .
$$

Now, by choosing

$$
x_{p}^{i}(t)=-\lambda(t),
$$

we get

$$
-\int_{0}^{T} \sum_{p \in \mathcal{P}^{i}}\left(\Psi_{p}^{i}\left(t, x^{*}(t)\right)+\alpha_{p}^{i}(t)-\beta^{i}(t)\right)^{2} d t \geq 0 .
$$


Therefore,

$$
\Psi_{p}^{i}\left(t, x^{*}(t)\right)-\beta^{i}(t)=-\alpha_{p}^{i}(t)
$$

Now, by (7) we obtain

$$
\left(\Psi_{p}^{i}\left(t, x^{*}(t)\right)-\beta^{i}(t)\right)\left(\bar{x}_{p}^{i}(t)-x_{p}^{* i}(t)\right)=0 .
$$

Conversely, let (3) and (4) be true and prove that (6) is verified. From (3) it follows that

$$
\sum_{p \in \mathcal{P}^{i}}\left(\Psi_{p}^{i}\left(t, x^{*}(t)\right)-\beta^{i}(t)\right)\left(x_{p}^{i}(t)-x_{p}^{* i}(t)\right) \geq 0
$$

In fact, if $x_{p}^{* i}(t)=0$ for a.e. $t \in[0, T]$, according to (3), it follows that $\Psi_{p}^{i}\left(t, x^{*}(t)\right)+\alpha_{p}^{i}(t)-\beta^{i}(t) \geq 0$ and $x_{p}^{i}(t)-x_{p}^{* i}(t) \geq 0$, hence (9) is verified. If $0<x_{p}^{* i}(t)<\bar{x}_{p}^{i}(t)$ for a.e. $t \in[0, T]$, by (3) it results that $\Psi_{p}^{i}\left(t, x^{*}(t)\right)+$ $\alpha_{p}^{i}(t)-\beta^{i}(t)=0$. Finally, if $x_{p}^{* i}(t)=\bar{x}_{p}^{i}(t)$ for a.e. $t \in[0, T], \Psi_{p}^{i}\left(t, x^{*}(t)\right)+$ $\alpha_{p}^{i}(t)-\beta^{i}(t) \leq 0$ and $x_{p}^{i}(t)-x_{p}^{* i}(t) \leq 0$, hence (9) is proved.

Since $\sum_{p \in \mathcal{P}_{(s, d)}^{i}} x_{p}^{i}(t)=\delta_{(s, d)}^{i}\left(t, x^{*}(t)\right)$ a.e. in $[0, T]$, we obtain

$$
\sum_{p \in \mathcal{P}^{i}} \Psi_{p}^{i}\left(t, x^{*}(t)\right)\left(x_{p}^{i}(t)-x_{p}^{* i}(t)\right) \geq 0
$$

and integrating we get

$$
\int_{0}^{T} \sum_{p \in \mathcal{P}^{i}} \Psi_{p}^{i}\left(t, x^{*}(t)\right)\left(x_{p}^{i}(t)-x_{p}^{* i}(t)\right) d t \geq 0 .
$$

Therefore, the proof is complete.

Remark 3 We observe that a combination of the above equilibrium behaviors, namely $x_{p}^{* i}(t)=0,0<x_{p}^{* i}(t)<\bar{x}_{p}^{i}(t)$ and $x_{p}^{* i}(t)=\bar{x}_{p}^{i}(t)$ a.e in $[0, T]$, can be performed to the end of examining a more general situation and describing all the fluctuating values of solutions during the time horizon.

Theorem $3 x^{* i}(t), i \in \mathcal{W}$, satisfies (5) if and only if it solves

$$
\begin{aligned}
& \int_{0}^{T} \sum_{p \in \mathcal{P}^{i}} C_{p}^{i}\left(t, x^{*}(t)\right)\left(x_{p}^{i}(t)-x_{p}^{* i}(t)\right) d t \geq 0, \\
& \forall x^{i}(t):\left(x^{1}(t), \ldots, x^{i}(t), \ldots, x^{W}(t)\right) \in K^{\mathcal{W}}\left(x^{*}\right) .
\end{aligned}
$$


Proof. The proof is on the lines of Theorem 2.1 in [16] and therefore will be omitted.

In order to express our equivalence result in a compact form, we introduce the vector $(F(t, x(t)))^{T}=(\Psi(t, x(t)), C(t, x(t))) \in L^{2}\left(0, T ; \mathbb{R}^{n+w}\right)$.

Theorem $4 x^{*}(t) \in K\left(x^{*}\right)$ is a mixed equilibrium if and only if it solves the following quasi-variational inequality

$$
\begin{aligned}
& \int_{0}^{T}\left\langle F\left(t, x^{*}(t)\right), x(t)-x^{*}(t)\right\rangle d t \\
& =\sum_{i \in \mathcal{N}} \int_{0}^{T} \sum_{p \in \mathcal{P}^{i}} \Psi_{p}^{i}\left(t, x^{*}(t)\right)\left(x_{p}^{i}(t)-x_{p}^{* i}(t)\right) d t \\
& +\sum_{i \in \mathcal{W}} \int_{0}^{T} \sum_{p \in \mathcal{P}^{i}} C_{p}^{i}\left(t, x^{*}(t)\right)\left(x_{p}^{i}(t)-x_{p}^{* i}(t)\right) d t \geq 0, \\
& \forall x(t) \in K\left(x^{*}\right) .
\end{aligned}
$$

Proof. From the preceding theorems it follows that if $x^{*}(t)=\left(x^{\mathcal{N}}(t), x^{\mathcal{W}}(t)\right)^{T} \in$ $K\left(x^{*}\right)$ is a mixed equilibrium, then $x^{\mathcal{N}}(t)=\left(x^{1}(t), \ldots, x^{i}(t), \ldots, x^{N}(t)\right)^{T}$ satisfies

$$
\sum_{i \in \mathcal{N}} \int_{0}^{T} \sum_{p \in \mathcal{P}^{i}} \Psi_{p}^{i}\left(t, x^{*}(t)\right)\left(x_{p}^{i}(t)-x_{p}^{* i}(t)\right) d t \geq 0,
$$

and $x^{\mathcal{W}}(t)=\left(x^{1}(t), \ldots, x^{i}(t), \ldots, x^{W}(t)\right)^{T}$ verifies

$$
\sum_{i \in \mathcal{W}} \int_{0}^{T} \sum_{p \in \mathcal{P}^{i}} C_{p}^{i}\left(t, x^{*}(t)\right)\left(x_{p}^{i}(t)-x_{p}^{* i}(t)\right) d t \geq 0 .
$$

Therefore, $x^{*}(t)$ solves (11) and the converse is also true.

\section{An existence result}

Before showing our result, for reader's convenience, we recall the following theorem (see [18]) adapted to our case.

Theorem 5 Let $X$ be a topological linear locally convex Hausdorff space and let $E \subset X$ be a convex, compact and nonempty subset. Let $F: E \rightarrow X^{\prime}$ be a continuous function and let $K: E \rightarrow 2^{E}$ be a closed lower semicontinuous multifunction with $K(x), x \in E$ convex, compact and nonempty. Then, there exists $x^{*} \in K\left(x^{*}\right)$

$$
\left\langle F\left(x^{*}\right), x-x^{*}\right\rangle \geq 0, \quad \forall x \in K\left(x^{*}\right) .
$$


Now we are able to prove the following result (see also $[15,16])$.

Theorem 6 Let us assume that a), c), d) and the following assumptions hold

e) $\exists \nu \in L^{2}(0, T), \nu(t) \geq 0$ for a.e. $t \in[0, T]$ :

$$
\left\|\delta\left(t, x_{1}\right)-\delta\left(t, x_{2}\right)\right\| \leq \nu(t)\left\|x_{1}-x_{2}\right\|, \quad \forall x_{1}, x_{2} \in \mathbb{R}^{n+w}
$$

f) $\Psi(t, x), C(t, x)$ and $\delta(t, x)$ are convex in $x$ for a.e. $t \in[0, T]$ and upper semicontinuous with respect to the weak topology in $x \in E$ for a.e. $t \in[0, T]$.

Then quasi-variational inequality problem (11) admits a solution.

Proof. The proof has recourse to weak topology arguments. First we observe that under hypotheses a), c), d) and if $x^{*}(t) \in L^{2}\left(0, T ; \mathbb{R}^{n+w}\right)$, it results that

$$
\begin{aligned}
& F\left(t, x^{*}(t)\right)=\left(\Psi\left(t, x^{*}(t)\right), C\left(t, x^{*}(t)\right)\right)^{T} \in L^{2}\left(0, T ; \mathbb{R}^{n+w}\right), \\
& \delta\left(t, x^{*}(t)\right) \in L^{2}\left(0, T ; \mathbb{R}^{m}\right) .
\end{aligned}
$$

Moreover, by a), c) and d) it follows that $F$ and $\delta$ belong to the class of Nemytskii operators, therefore if $x^{* k}(t) \stackrel{L^{2}}{\longrightarrow} x(t)$ then

$$
\left\|F\left(t, x^{* k}(t)\right)-F\left(t, x^{*}(t)\right)\right\|_{L^{2}} \rightarrow 0,\left\|\delta\left(t, x^{* k}(t)\right)-\delta\left(t, x^{*}(t)\right)\right\|_{L^{2}} \rightarrow 0,
$$

and the functions $F$ and $\delta$ are $L^{2}$-continuous with respect to the strong topology.

Now, in order to prove that $K\left(x^{*}\right)$ is a weakly closed multifunction, we show that it is strongly closed, i.e. for any sequences $\left\{x^{* k}(t)\right\}$ and $\left\{x^{k}(t)\right\}$ in $L^{2}(0, T)$ such that $x^{* k}(t) \stackrel{L^{2}}{\longrightarrow} x^{*}(t)$ and $x^{k}(t) \stackrel{L^{2}}{\longrightarrow} x(t)$, with $x^{k}(t) \in K\left(x^{* k}(t)\right) \forall k \in \mathbb{N}$, it results that $x(t) \in K\left(x^{*}\right)$.

Let $\left\{x^{* k}(t)\right\},\left\{x^{k}(t)\right\}$ two arbitrary convergent sequences in $L^{2}(0, T)$. Since $x^{k}(t) \in K\left(x^{* k}(t)\right)$, we have that $0 \leq x_{p}^{i k}(t) \leq \bar{x}_{p}^{i}(t)$, for a.e. $t \in[0, T]$, $\forall i \in \mathcal{I}, \forall p \in \mathcal{P}^{i}$ and, by the convergence of the sequence $\left\{x^{k}(t)\right\}$ in $L^{2}$, the bound constraints on $x(t)$ are satisfied. Moreover, $\forall i \in \mathcal{I}, \forall(s, d) \in D^{i}$, $\forall p \in \mathcal{P}_{(s, d)}^{i}$, it holds

$$
\sum_{p \in P_{(s, d)}^{i}} x_{p}^{i k}(t)=\delta_{(s, d)}^{i}\left(t, x^{* k}(t)\right) .
$$


The left-hand side converges almost everywhere to $\sum_{p \in P_{(s, d)}^{i}} x_{p}^{i}(t)$. By applying e) it follows that

$$
\left|\delta_{(s, d)}^{i}\left(t, x^{* k}(t)\right)-\delta_{(s, d)}^{i}\left(t, x^{*}(t)\right)\right| \longrightarrow 0,
$$

and thus we conclude that $x(t) \in K\left(x^{*}\right)$. Therefore, since $K\left(x^{*}(t)\right)$ is convex and strongly closed, we conclude that it is weakly closed.

In order to prove that $K\left(x^{*}\right)$ is a weakly lower semi-continuous setvalued map, we show that $\forall\left\{x^{* k}(t)\right\}$ weakly converging to $x^{*}(t)$ in $L^{2}$ (briefly $\left.x^{* k}(t) \rightarrow x^{*}(t)\right), \forall x(t) \in K\left(x^{*}\right)$ there exists $\left\{x^{k}(t)\right\}$ so that

$$
x^{k}(t) \rightarrow x(t) \text { with } x^{k}(t) \in K\left(x^{* k}(t)\right) \forall k \in \mathbb{N} .
$$

Let us consider an arbitrary sequence $x^{* k}(t) \rightarrow x^{*}(t), x(t) \in K\left(x^{*}\right)$ and fix $k \in \mathbb{N}, t \in[0, T]$. For all $i \in \mathcal{I}$ and $(s, d) \in D^{i}$ we introduce the following sets

$$
\begin{aligned}
A_{(s, d)}^{i} & =\left\{p \in \mathcal{P}_{(s, d)}^{i}\right\}, \\
B_{(s, d)}^{i} & =\left\{p \in A_{(s, d)}^{i}: \delta_{(s, d)}^{i}\left(t, x^{*}(t)\right)-\delta_{(s, d)}^{i}\left(t, x^{* k}(t)\right) \leq 0\right\}, \\
C_{(s, d)}^{i} & =\left\{p \in A_{(s, d)}^{i}: 0<\delta_{(s, d)}^{i}\left(t, x^{*}(t)\right)-\delta_{(s, d)}^{i}\left(t, x^{* k}(t)\right)<x_{p}^{i}(t)\right\}, \\
D_{(s, d)}^{i} & =\left\{p \in A_{(s, d)}^{i}: x_{p}^{i}(t) \leq \delta_{(s, d)}^{i}\left(t, x^{*}(t)\right)-\delta_{(s, d)}^{i}\left(t, x^{* k}(t)\right)\right\} .
\end{aligned}
$$

Let us also construct the sequence $x^{i k}(t)$, so that

$$
x_{p}^{i k}(t)= \begin{cases}x_{p}^{i}(t) & \text { if } p \in B_{(s, d)}^{i} \cup D_{(s, d)}^{i}, \\ x_{p}^{i k}(t)-\frac{\delta_{(s, d)}^{i}\left(t, x^{*}(t)\right)-\delta_{(s, d)}^{i}\left(t, x^{* k}(t)\right)}{\left|C_{(s, d)}^{i}\right|} & \text { if } p \in C_{(s, d)}^{i},\end{cases}
$$

If $p \in B_{(s, d)}^{i} \cup D_{(s, d)}^{i}, x_{p}^{i k}(t)=x_{p}^{i}(t)$ and, since $x(t) \in K\left(x^{*}\right)$, then the assertion is proved. If $p \in C_{(s, d)}^{i}$, it is easy to show that $x_{p}^{i k}(t)$ satisfies the bound constraints. 
Moreover,

$$
\begin{aligned}
\sum_{p \in \mathcal{P}_{(s, d)}^{i}} x_{p}^{i k}(t) & =\sum_{p \in A_{(s, d)}^{i}} x_{p}^{i k}(t)=\sum_{p \in B_{(s, d)}^{i} \cup D_{(s, d)}^{i}} x_{p}^{i k}(t) \\
& +\sum_{p \in C_{(s, d)}^{i}}\left(x_{p}^{i k}(t)-\frac{\delta_{(s, d)}^{i}\left(t, x^{*}(t)\right)-\delta_{(s, d)}^{i}\left(t, x^{* k}(t)\right)}{\left|C_{(s, d)}^{i}\right|}\right) \\
& =\sum_{p \in A_{(s, d)}^{i}} x_{p}^{i k}(t)-\left(\delta_{(s, d)}^{i}\left(t, x^{*}(t)\right)-\delta_{(s, d)}^{i}\left(t, x^{* k}(t)\right)\right) \\
& =\delta_{(s, d)}^{i}\left(t, x^{* k}(t)\right) .
\end{aligned}
$$

Therefore, demand requirements are verified and $x^{k}(t) \in K\left(x^{* k}(t)\right) \forall k \in \mathbb{N}$.

In order to prove that $\left\{x^{k}\right\}$ weakly converges to $x$, we show that

$$
\forall f(t) \in L^{2}(0, T), \lim _{k \rightarrow \infty} \int_{0}^{T} f(t)\left(x^{k}(t)-x(t)\right) d t=0 .
$$

Due to the construction of the sequence, we have

$$
\begin{aligned}
& \left|\int_{0}^{T} f(t)\left(x^{k}(t)-x(t)\right) d t\right|=\left|\int_{0}^{T} f(t) \sum_{i \in \mathcal{I}} \sum_{p \in A_{(s, d)}^{i}}\left(x_{p}^{i k}(t)-x_{p}^{i}(t)\right) d t\right| \\
& =\left|\int_{0}^{T} f(t) \sum_{i \in \mathcal{I}}\left[\sum_{p \in B_{(s, d)}^{i} \cup D_{(s, d)}^{i}}\left(x_{p}^{i k}(t)-x_{p}^{i}(t)\right)+\sum_{p \in C_{(s, d)}^{i}}\left(x_{p}^{i k}(t)-x_{p}^{i}(t)\right)\right] d t\right| \\
& =\left|\int_{0}^{T} f(t) \sum_{i \in \mathcal{I}} \sum_{p \in C_{(s, d)}^{i}}\left(\frac{\delta_{(s, d)}^{i}\left(t, x^{*}(t)\right)-\delta_{(s, d)}^{i}\left(t, x^{* k}(t)\right)}{\left|C_{(s, d)}^{i}\right|}\right) d t\right| \\
& =\left|\int_{0}^{T} f(t) \sum_{i \in \mathcal{I}}\left(\delta_{(s, d)}^{i}\left(t, x^{*}(t)\right)-\delta_{(s, d)}^{i}\left(t, x^{* k}(t)\right)\right) d t\right| .
\end{aligned}
$$

Now, we observe that $\delta$ is continuous with respect to the weak topology. In fact, on the one hand strong continuity and convexity of $\delta$ imply weak lower semicontinuity; on the other hand assumption $\mathrm{f}$ ) ensures weak upper semicontinuity. Therefore, the last expression of the above equality chain converges to zero and $x^{k}$ weakly converges to $x$. Moreover, as $K(x(t))$, $x(t) \in E$, is a convex, bounded and closed subset of $L^{2}\left(0, T ; \mathbb{R}^{n+w}\right)$, it is weakly compact (see Theorem B.4 in [10]). Finally, assumption f) and 
strong continuity of $\Psi$ and $C$ imply that $F$ is weakly continuous. Thus, all the assumptions of Theorem 4.1 are fulfilled with respect to the weak topology and the existence of at least one solution is ensured.

\section{$5 \quad$ Numerical example}

In order to support the theoretical results and illustrate the mixed equilibrium features, we consider the following network.

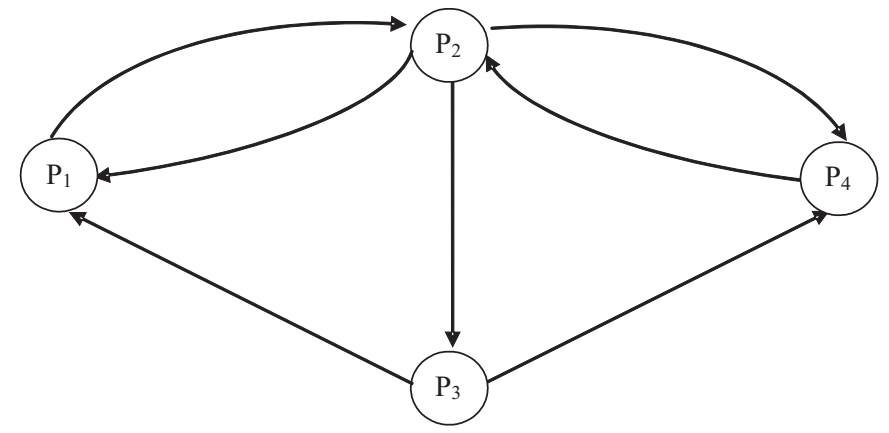

Figure 1: A network model example

We consider the time interval $[0, T]=[0,1]$. The origin-destination pairs are given by $\left(P_{1}, P_{4}\right)$ and $\left(P_{4}, P_{1}\right)$. We suppose that $\mathrm{O} / \mathrm{D}$ pair $\left(P_{1}, P_{4}\right)$ is controlled by a group user (Nash player), whereas $\mathrm{O} / \mathrm{D}$ pair $\left(P_{4}, P_{1}\right)$ is controlled by an individual user (Wardrop player). Therefore, the set of Nash players $\mathcal{N}$ is a singleton and the only element is also denoted by $\mathcal{N}$. Analogously, the set of Wardrop players is a singleton, whose only one element is denoted by $\mathcal{W}$.

Link flows used by the Nash player are

$$
\begin{array}{ll}
f_{1}^{\mathcal{N}}(t)=\left(P_{1}, P_{2}\right), & f_{2}^{\mathcal{N}}(t)=\left(P_{2}, P_{4}\right), \\
f_{3}^{\mathcal{N}}(t)=\left(P_{2}, P_{3}\right), & f_{4}^{\mathcal{N}}(t)=\left(P_{3}, P_{4}\right),
\end{array}
$$


and link flows used by the Wardrop player are

$$
\begin{array}{ll}
f_{1}^{\mathcal{W}}(t)=\left(P_{4}, P_{2}\right), & f_{2}^{\mathcal{W}}(t)=\left(P_{2}, P_{1}\right), \\
f_{3}^{\mathcal{W}}(t)=\left(P_{2}, P_{3}\right), & f_{4}^{\mathcal{W}}(t)=\left(P_{3}, P_{1}\right) .
\end{array}
$$

Hence, the total link flow is $f(t)=\left(f_{a}(t)\right)_{a=1, \ldots, 7}^{T}$, where

$$
\begin{array}{ll}
f_{1}(t)=f_{1}^{\mathcal{N}}(t), & f_{2}(t)=f_{2}^{\mathcal{N}}(t), \quad f_{3}(t)=f_{3}^{\mathcal{N}}(t)+f_{3}^{\mathcal{W}}(t), \\
f_{4}(t)=f_{4}^{\mathcal{N}}(t), & f_{5}(t)=f_{1}^{\mathcal{V}}(t), \quad f_{6}(t)=f_{2}^{\mathcal{W}}(t), \quad f_{7}(t)=f_{4}^{\mathcal{W}}(t) .
\end{array}
$$

It follows immediately that the Nash player controls the paths

$$
p_{1}=\left(P_{1}, P_{2}\right) \cup\left(P_{2}, P_{4}\right), \quad p_{2}=\left(P_{1}, P_{2}\right) \cup\left(P_{2}, P_{3}\right) \cup\left(P_{3}, P_{4}\right),
$$

whereas the Wardrop player controls the paths

$$
p_{3}=\left(P_{4}, P_{2}\right) \cup\left(P_{2}, P_{1}\right), \quad p_{4}=\left(P_{4}, P_{2}\right) \cup\left(P_{2}, P_{3}\right) \cup\left(P_{3}, P_{1}\right) .
$$

The following link cost functions are given (see [9] for the static version of the model)

$$
\begin{array}{ll}
c_{1}(f(t))=20+f_{1}(t), & c_{2}(f(t))=20+f_{2}(t), \quad c_{3}(f(t))=5+2 f_{3}(t), \\
c_{4}(f(t))=5+2 f_{4}(t), & c_{5}(f(t))=20+f_{1}(t), \quad c_{6}(f(t))=20+f_{2}(t), \\
c_{7}(f(t))=5+2 f_{4}(t) . &
\end{array}
$$

We denote by $x(t)=\left(x_{1}(t), x_{2}(t), x_{3}(t), x_{4}(t)\right)^{T}$ the path flow vector, where

$$
\begin{array}{ll}
x_{1}(t)=x_{p_{1}}^{\mathcal{N}}(t), & x_{2}(t)=x_{p_{2}}^{\mathcal{N}}(t), \\
x_{3}(t)=x_{p_{3}}^{\mathcal{W}}(t), & x_{4}(t)=x_{p_{4}}^{\mathcal{W}}(t),
\end{array}
$$

$x_{p_{r}}^{\mathcal{N}}(t), r=1,2$, (respectively, $\left.x_{p_{r}}^{\mathcal{W}}(t), r=3,4\right)$ is the flow on path $p_{r}$ generated by users of the set $\mathcal{N}$ (respectively $\mathcal{W}$ ). We now introduce the cost 
functions of users

$$
\begin{aligned}
J^{\mathcal{N}}(x(t)) & =\sum_{r=1}^{2} x_{p_{r}}^{\mathcal{N}}(t) \sum_{a=1}^{7} \gamma_{a p_{r}} \frac{1}{\mu_{a}(t)} c_{a}(f(t)), \\
C_{p_{r}}^{\mathcal{W}}(x(t)) & =\sum_{a=1}^{7} \gamma_{a p_{r}} \frac{1}{\mu_{a}(t)} c_{a}(f(t)), \quad r=3,4 .
\end{aligned}
$$

For simplicity we fix $\mu_{a}(t)=1, \forall a \in \mathcal{L}$ a.e. in $[0,1]$. Introduced the notations

$$
\begin{aligned}
& \Psi(x(t))=\left(\begin{array}{c}
\frac{\partial J^{\mathcal{N}}(x)}{\partial x_{p_{1}}^{\mathcal{N}}} \\
\frac{\partial J^{\mathcal{N}}(x)}{\partial x_{p_{2}}^{\mathcal{N}}}
\end{array}\right), \\
& C(x(t))=\left(\begin{array}{c}
C_{p_{3}}^{\mathcal{W}}(x(t)) \\
C_{p_{4}}^{\mathcal{W}}(x(t))
\end{array}\right),
\end{aligned}
$$

we introduce the vector $F$

$$
F(x(t))=\left(\begin{array}{c}
\Psi(x(t)) \\
C(x(t))
\end{array}\right),
$$

whose components are given by

$$
\begin{aligned}
& F_{1}(x(t))=4 x_{1}(t)+2 x_{2}(t)+40, \\
& F_{2}(x(t))=2 x_{1}(t)+10 x_{2}(t)+4 x_{4}(t)+30, \\
& F_{3}(x(t))=2 x_{3}(t)+x_{4}(t)+40 \\
& F_{4}(x(t))=2 x_{2}(t)+x_{3}(t)+5 x_{4}(t)+30 .
\end{aligned}
$$

The equilibrium flow is a solution to the quasi-variational inequality

$$
\text { Find } x^{*} \in K\left(x^{*}\right): \int_{0}^{1}\left\langle F\left(x^{*}(t)\right), x(t)-x^{*}(t)\right\rangle d t \geq 0, \quad \forall x(t) \in K\left(x^{*}\right),
$$

where

$$
\begin{aligned}
K\left(x^{*}(t)\right)=\quad & \left\{x(t) \in L^{2}\left(0,1 ; \mathbb{R}^{4}\right): x_{i}(t) \geq 0, i=1, \ldots, 4\right. \\
& x_{1}(t)+x_{2}(t)=\delta_{\left(P_{1}, P_{4}\right)}^{\mathcal{N}}, x_{3}(t)+x_{4}(t)=\delta_{\left(P_{4}, P_{1}\right)}^{\mathcal{W}} \\
& \text { a.e. in }[0, T]\} .
\end{aligned}
$$


and

$$
\begin{aligned}
& \delta_{\left(P_{1}, P_{4}\right)}^{\mathcal{N}}\left(t, x^{*}(t)\right)=10 t+\frac{2}{3} x_{1}^{*}(t)+1, \\
& \delta_{\left(P_{4}, P_{1}\right)}^{\mathcal{W}}\left(t, x^{*}(t)\right)=4 t+\frac{1}{2} x_{4}^{*}(t)+3 .
\end{aligned}
$$

After some calculations we obtain the exact solution

$$
\left(\frac{888}{55} t+\frac{21}{11}, \frac{254}{55} t+\frac{4}{11}, \frac{252}{55} t+\frac{18}{11},-\frac{64}{55} t+\frac{30}{11}\right) .
$$

It is worth noting that player controlling $\mathrm{O} / \mathrm{D}$ pair $\left(P_{1}, P_{4}\right)$ acts in a system equilibrium way, but the system is not in a system equilibrium. In fact the system equilibrium, obtained solving (12) with

$$
\begin{aligned}
& F_{1}\left(x^{*}(t)\right)=4 x_{1}^{*}(t)+2 x_{2}^{*}(t)+40, \\
& F_{2}\left(x^{*}(t)\right)=2 x_{1}^{*}(t)+10 x_{2}^{*}(t)+4 x_{4}^{*}(t)+30, \\
& F_{3}\left(x^{*}(t)\right)=4 x_{3}^{*}(t)+2 x_{4}^{*}(t)+40, \\
& F_{4}\left(x^{*}(t)\right)=4 x_{2}^{*}(t)+2 x_{3}^{*}(t)+10 x_{4}^{*}(t)+30,
\end{aligned}
$$

is given by

$$
\left(\frac{888}{55} t+\frac{9}{11}, \frac{254}{55} t+\frac{8}{11}, \frac{252}{55} t+\frac{25}{11},-\frac{64}{55} t+\frac{16}{11}\right) .
$$

Under regularity assumptions, solutions are continuous, hence we may numerically solve a sequence of finite-dimensional quasi-variational inequality problems

$$
\left\langle F\left(x\left(t_{n}^{j}\right)\right), x-x\left(t_{n}^{j}\right)\right\rangle \geq 0, \quad \forall x \in K\left(x\left(t_{n}^{j}\right)\right),
$$

where a discrete approximation of the time variable is performed by means of a sequence of partitions $\pi_{n}=0=t_{n}^{0}<t_{n}^{1}<\ldots<t_{n}^{N_{n}}=T$ of the time interval $[0,1]$. We denote by $k_{n}^{j}=t_{n}^{j}-t_{n}^{j-1}$ the step-size, for $j=1, \ldots, N_{n}$ and set $k_{n}=\max _{j} k^{j}$.

We omit the statements of the example, solved by applying the extragradient method (see $[3,13,12,11]$ for a discussion on computational procedures), with $N_{n}=30$, and directly provide numerical results in graphical form, see Figure 2. 



Figure 2: Curves of equilibria 
The results are, in some sense, paradoxical. In fact, in the examined situation, player $\left(P_{1}, P_{4}\right)$ acts as the player with market power, whereas player $\left(P_{4}, P_{1}\right)$ acts as a price-taker. Therefore one would expect that player $\left(P_{1}, P_{4}\right)$ would have a lower cost than player $\left(P_{4}, P_{1}\right)$. Surprisingly, player $\left(P_{4}, P_{1}\right)$ takes advantage from the market power of player $\left(P_{1}, P_{4}\right)$ so that he incurs in a lower cost than player $\left(P_{1}, P_{4}\right)$.

\section{Conclusions}

In this paper we presented time-dependent routing games in telecommunication networks shared by users with different market power. We studied the associated mixed equilibria in the framework of the variational inequality theory. In particular, users are assumed to incur in equilibrium-dependent travel demands, so that the model, to the best of our knowledge for the first time, was described by a quasi-variational inequality.

This paper aimed at improving previous literature on this topic, see $[4,9]$, taking into consideration the evolution of the system with respect to time and hence including in the model all the route choices experienced by users during the whole time interval.

Future extensions of the work include the following issues. First, the model can be refined by introducing a memory term, which shows how the current equilibrium situation is affected by the equilibrium distribution of the previous observation times. Second, the mixed equilibrium can be approached by duality theory, so that new perspectives and interpretations of the model can be unveiled.

\section{References}

[1] E. Altman, T. Boulogne, R. El-Azouzi, T. Jimnez and L. Wynter, A survey on networking games in telecommunications, Computers and Operations Research, Vol. 33 (2), 2006, 286-311.

[2] C. Baiocchi and A. Capelo, Variational and Quasivariational Inequalities. Applications to Free Boundary Problems, J. Wiley and Sons, New York, 1984.

[3] M. Bliemer and P. Bovy, Quasi-Variational inequality formulation of the multiclass dynamic traffic assignment problem, Transportation Research, B37 (2003), 501-519. 
[4] T. Boulogne, E. Altman, H. Kameda and O. Pourtallier, Mixed Equilibrium (ME) for Multiclass Routing Games, IEEE Transaction on Automatic Control, 47 (2002), no. 6, 903-916.

[5] P. Daniele, Lagrangean Function for Dynamic Variational Inequalities, Rendiconti del Circolo Matematico di Palermo, Serie II, Suppl., 58 (1999), 101-119.

[6] P. Daniele, F. Giannessi, and A. Maugeri (Eds.), "Equilibrium Problems and Variational Models", Kluwer Academic Publishers, 2003.

[7] F. Giannessi and A. Maugeri (Eds.), "Variational Inequalities and Network Equilibrium Problems", Plenum Publishing, New York, 1995.

[8] F. Giannessi, A. Maugeri and P. Pardalos (Eds.), "Equilibrium Problems: Non- smooth Optimization and Variational Inequality Models", Kluwer Academic Publishers, Dordrecht, The Netherlands, 2001.

[9] P.T. Harker, Mixed Equilibrium Behaviours on Networks, Transportation Science, 22 (1988), no. 1, 39-46.

[10] J. Jahn, Introduction to the Theory of Nonlinear Optimization, Springer- Verlag, Berlin, Germany, 1996.

[11] Yu. Nesterov and L. Scrimali, Solving strongly monotone variational and quasi-variational inequalities, CORE DP 2006/107.

[12] Yu. Nesterov, Dual extrapolation and its applications to solving variational inequalities and related problems, CORE DP 2003/68. Accepted by Mathematical Programming.

[13] P. Marcotte, Applications of Khobotov's algorithm to variational inequalities and network equilibrium problems, Inform. Systems Oper. Res., 29 (1991), 258-270.

[14] A. Maugeri, W. Oettli and D. Schläger, A Flexible Form of Wardrop's Principle for Traffic Equilibria with Side Constraints, Supplemento Rendiconti del Circolo Matematico di Palermo, Serie 248 (1997), 185193.

[15] F. Raciti, and L. Scrimali, Time-dependent variational inequalities and applications to equilibrium problems, Journal of Global Optimization, 28 (2004), no. 4, 387-400. 
[16] L. Scrimali, Quasi-variational Inequalities in Transportation Networks, Mathematical Models and Methods in Applied Sciences, 14 (2004), no. 10, 1541-1560.

[17] G. Stampacchia, Variational Inequalities, Theory and Applications of Monotone Operators, Proceedings of a NATO Advanced Study Institute (Venice, 1968), Oderisi, Gubbio, 101-192, 1969.

[18] N. X. Tan, Quasi-Variational Inequality in Topological Linear Locally Convex Hausdorff Spaces, Math. Nachr. 122 (1985), 231-245.

[19] J.G. Wardrop, Some Theoretical Aspects of Road Traffic Research, Proceedings of the Institute of Civil Engineers, Part II, 325-378, 1952.

[20] B. W. Wie, A differential game approach to the dynamic mixed behaviour traffic network equilibrium problem, European Journal of Operational Research, 83 (1995), 117-136. 